\title{
National Estimates of the Quantity and Cost of Informal Caregiving for the Elderly with Dementia
}

\author{
Kenneth M. Langa, MD, PhD, Michael E. Chernew, PhD, Mohammed U. Kabeto, MS, \\ A. Regula Herzog, PhD, Mary Beth Ofstedal, PhD, Robert J. Willis, PhD, Robert B. Wallace, MD, \\ Lisa M. Mucha, PhD, Walter L. Straus, MD, MPH, A. Mark Fendrick, MD
}

OBJECTIVE: Caring for the elderly with dementia imposes a substantial burden on family members and likely accounts for more than half of the total cost of dementia for those living in the community. However, most past estimates of this cost were derived from small, nonrepresentative samples. We sought to obtain nationally representative estimates of the time and associated cost of informal caregiving for the elderly with mild, moderate, and severe dementia.

DESIGN: Multivariable regression models using data from the 1993 Asset and Health Dynamics Study, a nationally representative survey of people age 70 years or older $(N=7,443)$.

SETTING: National population-based sample of the community-dwelling elderly.

MAIN OUTCOME MEASURES: Incremental weekly hours of informal caregiving and incremental cost of caregiver time for those with mild dementia, moderate dementia, and severe dementia, as compared to elderly individuals with normal cognition. Dementia severity was defined using the Telephone Interview for Cognitive Status.

RESULTS: After adjusting for sociodemographics, comorbidities, and potential caregiving network, those with normal cognition received an average of 4.6 hours per week of informal care. Those with mild dementia received an additional 8.5 hours per week of informal care compared to those with normal cognition $(P<.001)$, while those with moderate and severe dementia received an additional 17.4 and 41.5 hours

Received from the Division of General Medicine, Department of Medicine (KML, MEC, AMF), the Institute for Social Research (KML, ARH, MBO, RJW), the Consortium for Health Outcomes, Innovation, and Cost-Effectiveness Studies (CHOICES) (MEC, MUK, AMF), and the Department of Health Management and Policy, School of Public Health (MEC, AMF), University of Michigan, Ann Arbor, Mich; the VA Center for Practice Management and Outcomes Research (KML), Ann Arbor, Mich; the Colleges of Public Health and Medicine, University of Iowa (RBW), Iowa City, Iowa; and Outcomes Research and Management, Merck and Company, Inc., (LMM, WLS), West Point, Pa.

An early version of this work was presented at the annual meeting of the Society of General Internal Medicine, May 5, 2000, Boston, Mass.

Address correspondence and reprint requests to Dr. Langa: University of Michigan Health System, Division of General Medicine, 300 North Ingalls Building, Room 7E01, Box 0429, Ann Arbor, MI 48109-0429 (e-mail: klanga@umich.edu).
$(P<.001)$, respectively. The associated additional yearly cost of informal care per case was $\$ 3,630$ for mild dementia, $\$ 7,420$ for moderate dementia, and $\$ 17,700$ for severe dementia. This represents a national annual cost of more than $\$ 18$ billion.

CONCLUSION: The quantity and associated economic cost of informal caregiving for the elderly with dementia are substantial and increase sharply as cognitive impairment worsens. Physicians caring for elderly individuals with dementia should be mindful of the importance of informal care for the well-being of their patients, as well as the potential for significant burden on those (often elderly) individuals providing the care.

KEY WORDS: informal caregiving; Alzheimer's disease; dementia; chronic disease; health economics. J GEN INTERN MED 2001;16:770-778.

D ementia, a deterioration in intellectual capacity that impairs independent functioning in everyday life, is a common condition affecting an estimated $5 \%$ to $10 \%$ of Americans aged 65 and over. $^{1}$ In the United States, the prevalence of Alzheimer's disease ( $\mathrm{AD}$ ), the most common cause of dementia, is projected to more than triple (from about 2.3 to 8.7 million cases) in the next 50 years due to aging of the population. ${ }^{2}$ This large increase in the prevalence of dementia will generate substantial additional burdens on the formal health care system and on the network of informal caregivers that typically provides most of the daily care required by individuals with dementia. Primary care physicians, because of their key role in the long-term management of elderly individuals with chronic disease, will be disproportionately affected by the growing prevalence of dementia among their patients, as well as the growing caregiving burden among their patients' spouses and children.

Most studies have found that the cost associated with unpaid informal caregiver time accounts for a majority of the total cost of dementia, ${ }^{3}$ due to the progressive functional limitations in activities of daily living (ADLs) and instrumental activities of daily living (IADLs) that are the hallmark of the disease. However, the estimates of the average caregiver time and associated cost for dementia care have varied widely (from $\$ 1,500^{4}$ to $\$ 35,000^{5}$ per year), probably due to the use of small geographically restricted samples as 
well as differences across studies in average severity of cognitive impairment, definitions and methods for establishing a dementia diagnosis, and the "wage" or "opportunity cost" used to value informal caregiver time. ${ }^{4-13}$

Only one prior study ${ }^{7}$ used a nationally representative U.S. sample to evaluate informal caregiving for dementia, but that study did not adjust for important socioeconomic and family characteristics that have been shown to be important independent predictors of informal care.

Our objective was to obtain more valid and generalizable estimates of the time and associated cost of informal caregiving for the elderly with different levels of cognitive impairment by using a population-based nationally representative sample of the community-dwelling elderly age 70 years or older. In addition, we aimed to better identify the incremental or additional caregiving attributable to dementia, net of the caregiving that results due to coexisting chronic diseases, and to better adjust for differences in important sociodemographic and family characteristics that might independently affect provision of informal care. By estimating a nationally representative cost of informal caregiving for various stages of dementia, we hope to provide useful data for future evaluations of the social and economic impact of the increasing number of elderly with dementia, as well as the increasing number of interventions ${ }^{14,15}$ aimed at decreasing dementia incidence and progression.

\section{METHODS}

\section{Conceptual Model of Informal Caregiving for Dementia}

The conceptual model of informal caregiving underlying our analysis is shown in Figure 1. We assume that brain pathology (e.g., the accumulation of $\beta$-amyloid plaques in $\mathrm{AD}$ or strokes in multi-infarct dementia) leads to the progressive memory and other cognitive impairments of dementia. These cognitive impairments result in limitations in the independent performance of ADLs and IADLs. ${ }^{16}$ The majority of informal care is provided to address these functional limitations, although additional care is provided to manage behavioral problems associated with dementia, such as psychiatric symptoms and wandering, $5,10,13$ and to provide supervision for safety. ${ }^{13}$ Elderly individuals with dementia may also have comorbid conditions (e.g., diabetes, heart disease, arthritis, and cancer) that lead to functional limitations independently of their cognitive impairment. Certain complications of dementia, such as increased risk of falls or urinary incontinence, may result in further ADL or IADL difficulties in addition to those resulting directly from cognitive impairment. Finally, other variables, such as an individual's age, gender, race, socioeconomic status, the relative availability of informal caregivers, and the availability of possible substitutes for informal care (e.g., paid home care and nursing home services) may also affect the total amount of care provided by informal caregivers. ${ }^{17-20}$

Properly identifying and attributing the caregiving costs that arise from a particular disease may be difficult. $^{21,22}$ Consider, for instance, an elderly individual with dementia who also has diabetes. The informal caregiving that results from diabetic complications such as the visual impairment of retinopathy should not be attributed to the presence of dementia since the impairment is not directly due to dementia. However, compared to an individual with normal cognitive function, a given level of visual impairment in an individual with dementia may result in even greater caregiving needs (e.g., due to

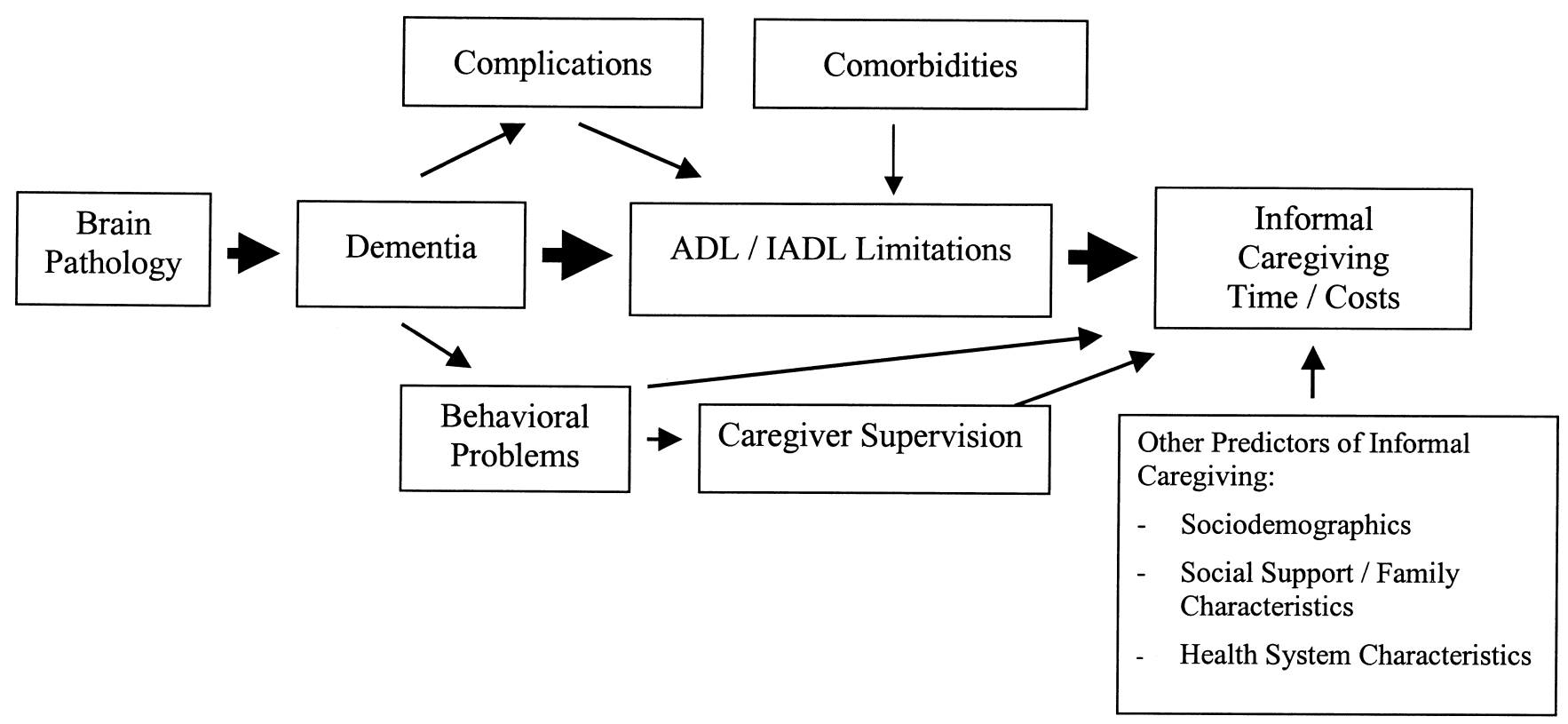

FIGURE 1. Conceptual model of informal caregiving for dementia. 
difficulty in understanding and following directions) and, therefore, some portion of caregiving costs for the diabetic complication might be properly attributed to dementia. Likewise, some falls and resulting trauma are likely due to the orientation and attention difficulties associated with dementia, ${ }^{23,24}$ but a significant number of falls occur in elderly individuals with no cognitive impairment. So a portion of fall-related caregiving might be properly attributed to dementia in some elderly, but not in others.

Two methods have been used in prior studies to address the issue of correctly attributing informal caregiving costs to dementia. One study directly asked caregivers to identify only those helping tasks that resulted from an individual's dementia. ${ }^{5}$ Other studies used statistical modeling to determine the "net" caregiving attributable to dementia after adjusting for the presence of coexisting chronic conditions. ${ }^{7,9,12}$ Our analysis used a statistical modeling methodology, as described below, that is similar to this latter group of studies.

\section{Data}

We used data from the baseline 1993 survey of the Asset and Health Dynamics (AHEAD) Study, a biennial longitudinal survey of a nationally representative cohort of the U.S. elderly born in 1923 or earlier. ${ }^{25}$ Our analysis included all 7,443 community-dwelling elderly age 70 years or older who were enrolled in the baseline survey. This sample is representative of the approximately 21 million community-dwelling elderly in the United States.

The AHEAD survey was designed to study health transitions in old age and their impact on individuals, families, and society. In addition to measures of the health and functional status of the elderly survey respondents, data are collected on the number of hours of care provided by both paid and unpaid caregivers in the home. Most respondents age 70 to 79 years $(72 \%)$ were interviewed by telephone, while most of those age 80 years and older $(70 \%)$ were interviewed in person. The overall survey response rate was $80 \%$, and response rate did not differ significantly for those interviewed by phone compared to those interviewed in person. ${ }^{25}$

Approximately $10 \%$ of respondents were unable or unwilling to complete the AHEAD survey by themselves. ${ }^{25}$ A proxy respondent, most often a spouse (45\%) or daughter (29\%), completed the survey for these individuals.

The AHEAD Study was approved by the Behavioral Sciences Committee institutional review board at the University of Michigan. The data used for this analysis contained no unique identifiers, so respondent anonymity was maintained.

\section{Dependent Variables}

We classified respondents as receiving informal care if in-home assistance with any ADL (eating, transferring, toileting, dressing, bathing, walking across a room) or IADL (preparing meals, grocery shopping, making phone calls, taking medications, managing money) was provided by a relative (paid or not), or unpaid nonrelative with no organizational affiliation. ${ }^{26}$ AHEAD respondents were identified as recipients of informal care if, because of a health problem, they received any help with IADLs. However, due to the survey design, we were able to identify only those respondents who received help with an $\mathrm{ADL}$ "most of the time." So infrequent help for ADLs (that provided "some of the time" or "occasionally") was not included in the analysis.

The intensity (number of weekly hours) of informal home care was calculated using the average number of days per week (in the prior month), and average number of hours per day that respondents reported receiving help from informal caregivers. The methodology used for calculating weekly hours of care from the AHEAD data has been previously described. ${ }^{19}$ Because data on hours per day of care were not collected for caregivers who helped less than once per week, hours values for these caregivers were assigned using a method of multiple imputation ${ }^{27}$ based on reported caregiver characteristics (helper gender, residential status, relationship to the respondent, and number of days per week of care). ${ }^{19}$ Missing caregiving hours data were imputed for a total of 19\% of informal caregivers. Because most caregivers with missing data were those who provided very infrequent help (less than once per week), they accounted for less than $2 \%$ of the total informal caregiver hours analyzed in the study. We re-ran all analyses after dropping any observation for which data were imputed and found no significant change in our results. We imposed a limit of 16 hours of care per day for any individual caregiver to allow for 8 hours of sleep. ${ }^{8,28}$ This truncation of caregiving hours affected about $6 \%$ of the 2,700 informal caregivers identified in the AHEAD survey.

\section{Definitions of Dementia and Dementia Severity}

For self-respondents, the presence and severity of dementia were defined using a modified version of the Telephone Interview for Cognitive Status (TICS), a validated cognitive screening instrument patterned on the MiniMental State Examination (MMSE), ${ }^{29}$ which is specifically designed for population-based studies. ${ }^{30-32}$ On the basis of prior validation studies, we defined a cut-off score of 8 (2 standard deviations below the mean) on the 35-point TICS scale as the level of cognitive impairment consistent with "dementia." ${ }^{31-33}$ Further, severity of dementia was categorized using the TICS score as follows: a score of 6 to 8 was defined as "mild" dementia; 4 or 5 was defined as "moderate" dementia; and 0 to 3 was defined as "severe" dementia.

The TICS was not administered to those respondents represented by a proxy, but each proxy was asked: "How would you rate (the respondent's) memory at the present time?" and "How would you rate (the respondent) in making judgements and decisions?" The possible responses to each question were: "excellent," "very good," "good," "fair," or 
"poor." If a respondent's memory was assessed as "excellent," "very good," or "good" they were considered to have "normal" cognitive function, while those with "fair" or "poor" memory were considered to have cognitive impairment consistent with dementia. Further, for those proxy respondents with dementia, severity was assigned on the basis of proxy responses to the "judgement" question: those with "excellent," "very good," or "good" judgement were assigned to the "mild" dementia category, those with "fair" judgement were assigned to the "moderate" dementia category, and those with "poor" judgement were assigned to the "severe" dementia category. We performed sensitivity analyses using alternative cut-off scores for self-respondent dementia severity classification, and alternative proxy assessment categorizations to determine if the caregiving results changed significantly.

\section{Independent Variables and Statistical Model for Identifying Dementia Caregiving}

Since the goal of the analysis was to identify the additional informal caregiving attributable to dementia, we constructed a multivariable regression model that included the following independent variables: 1) categorical variables indicating the presence of mild, moderate, or severe dementia; 2) categorical variables indicating the presence of each of the following comorbidities: stroke, diabetes, heart disease, hypertension, lung disease, cancer, psychiatric problem, and arthritis; 3) categorical variables indicating the availability of potential informal caregivers (whether a spouse was present and whether the individual had a living child); and 4) sociodemographic characteristics (age, race, gender, net worth). We defined net worth terciles (low, middle, and high) using the full 1993 AHEAD sample.

To test the hypothesis that the presence of dementia results in additional caregiving for other comorbidities (e.g., caregiving to address diabetic complications is greater for diabetics with dementia than for diabetics with normal cognitive function), interaction terms for each of the comorbidities (e.g., dementia severity $\times$ diabetes) were tested in the regression model. None of these interaction terms were statistically significant, so they were not included in the final regression model.

In the resulting statistical model, the regression coefficients for the dementia severity variables indicate the additional caregiving associated with mild, moderate, and severe dementia (compared to those with normal cognitive function), after adjusting for differences across these four groups (normal, mild, moderate, severe) in the presence of each of the comorbidities, availability of informal caregivers, and sociodemographic characteristics. As such, the statistical model identifies the incremental caregiving attributable to dementia, net of differences in caregiving due to the presence of comorbidities and taking into account other differences between groups. ${ }^{7,12}$

The number of ADL and IADL limitations was not controlled for in the regression model since, as shown in
Figure 1, we hypothesize (on the basis of prior studies $^{5,10,13}$ ) that the majority of informal care provided for those with dementia is due to differences in ADL/IADL limitations in those with dementia, as compared to those with normal cognitive function.

Due to lack of data, we were unable to determine the amount of caregiving provided for supervision and behavior management. We were also unable to test the effect of health system characteristics (e.g., availability of paid home care, availability of nursing homes, enrollment in managed care systems) on the amount of informal care provided for those with dementia.

Because a substantial proportion of respondents received no informal care in the month prior to the administration of the survey, and the distribution of hours among recipients was highly skewed, we analyzed caregiving using a standard two-part multivariable model. ${ }^{18,34}$ For ease of interpretation, regression results from the two-part model were retransformed from log hours to hours. ${ }^{35}$

\section{Calculating the Cost of Informal Care}

Opportunity cost, or the value of a resource in its next best use to society, is the preferred measure of cost for economic analyses in health care. ${ }^{21}$ The opportunity cost of an informal caregiver's time is sometimes assigned using the average hourly wage of working individuals with similar characteristics (age, gender, education), but for some groups of caregivers (the retired elderly, for instance) there are no appropriate wage data. An alternate approach is to use the market price of an equivalent service (such as a home health aide) as an estimate of the opportunity cost of a caregiver's time. ${ }^{21}$ Using this latter method, we estimated the yearly cost of family caregiving for each level of cognitive impairment by multiplying the 1998 national average wage for a home health aide ( $\$ 8.20$ per hour ${ }^{36}$ ) by the adjusted weekly hours of care, and then multiplying by 52 (weeks per year). We performed a sensitivity analysis using the 10th percentile home health aide wage rate $(\$ 5.90$ per hour) as a more conservative estimate of the opportunity cost of caregiver time, and the 90th percentile wage rate (\$10.80 per hour) as a more generous estimate in order to provide a reasonable range of imputed family caregiving costs.

All analyses were weighted and adjusted for the complex sampling design (stratification, clustering, and nonresponse) of the AHEAD survey. ${ }^{25,37}$ STATA Statistical Software, Release 6.0, (Stata Corp, College Station, Tex) was used for data analysis. ${ }^{37}$ All reported $P$ values are twotailed, and a $P$ value $<.05$ was considered statistically significant.

\section{RESULTS}

\section{Characteristics of the Study Population}

The characteristics of those age 70 years or older in the 1993 AHEAD survey $(N=7,443)$ are shown in Table 1 . Ten 
Table 1. Characteristics of the Study Population, by Dementia Severity $(N=7,443)$

\begin{tabular}{|c|c|c|c|c|}
\hline \multirow[b]{2}{*}{ Variable } & \multicolumn{4}{|c|}{ Weighted Percentage* } \\
\hline & $\begin{array}{c}\text { Normal } \\
\text { Cognition } \\
(n=6,639)\end{array}$ & $\begin{array}{c}\text { Mild } \\
\text { Dementia } \\
(n=345)\end{array}$ & $\begin{array}{l}\text { Moderate } \\
\text { Dementia } \\
(n=212)\end{array}$ & $\begin{array}{c}\text { Severe } \\
\text { Dementia } \\
(n=247)\end{array}$ \\
\hline \multicolumn{5}{|l|}{ Age, $y^{\dagger}$} \\
\hline $70-79$ & 70 & 46 & 44 & 36 \\
\hline $80-89$ & 27 & 47 & 41 & 42 \\
\hline$\geq 90$ & 3 & 7 & 15 & 22 \\
\hline \multicolumn{5}{|l|}{ Race $^{\dagger}$} \\
\hline White & 89 & 70 & 72 & 72 \\
\hline African American & 9 & 25 & 24 & 25 \\
\hline Other & 2 & 5 & 3 & 4 \\
\hline \multicolumn{5}{|l|}{ Gender } \\
\hline Male & 38 & 41 & 45 & 35 \\
\hline Female & 62 & 59 & 55 & 65 \\
\hline \multicolumn{5}{|l|}{ Education, $\mathrm{y}^{\dagger}$} \\
\hline$<12$ & 40 & 73 & 74 & 63 \\
\hline 12 & 32 & 16 & 17 & 23 \\
\hline$\geq 13$ & 28 & 11 & 9 & 14 \\
\hline \multicolumn{5}{|l|}{ Net worth, $\mathbf{S}^{\dagger}$} \\
\hline$<38,000$ & 28 & 51 & 50 & 60 \\
\hline $38,000-139,000$ & 34 & 33 & 29 & 23 \\
\hline$>139,000$ & 38 & 16 & 21 & 17 \\
\hline \multicolumn{5}{|l|}{$\begin{array}{l}\text { Potential caregiver } \\
\text { network }\end{array}$} \\
\hline Spouse present ${ }^{\dagger}$ & 50 & 44 & 44 & 40 \\
\hline Living child & 90 & 91 & 87 & 89 \\
\hline \multicolumn{5}{|l|}{ ADLs impaired, $n^{\dagger}$} \\
\hline 0 & 74 & 50 & 36 & 20 \\
\hline $1-3$ & 22 & 33 & 41 & 31 \\
\hline $4-6$ & 4 & 17 & 23 & 48 \\
\hline \multicolumn{5}{|l|}{ IADLs impaired, $n^{\dagger}$} \\
\hline 0 & 75 & 39 & 21 & 8 \\
\hline $1-3$ & 24 & 51 & 54 & 28 \\
\hline $4-5$ & 2 & 10 & 25 & 64 \\
\hline \multicolumn{5}{|l|}{ Chronic conditions } \\
\hline Stroke $^{\dagger}$ & 9 & 18 & 19 & 37 \\
\hline Diabetes $^{\dagger}$ & 12 & 14 & 16 & 14 \\
\hline Heart disease $^{\dagger}$ & 31 & 32 & 40 & 34 \\
\hline Hypertension & 50 & 48 & 46 & 54 \\
\hline Lung disease & 12 & 13 & 10 & 10 \\
\hline Cancer & 14 & 12 & 11 & 12 \\
\hline \multicolumn{5}{|l|}{ Psychiatric } \\
\hline problem $^{\dagger}$ & 10 & 10 & 20 & 16 \\
\hline Arthritis ${ }^{\dagger}$ & 25 & 29 & 38 & 18 \\
\hline \multicolumn{5}{|l|}{ Respondent type $^{\dagger}$} \\
\hline Self & 94 & 71 & 44 & 18 \\
\hline Proxy & 6 & 29 & 56 & 82 \\
\hline
\end{tabular}

* Weighted percentage derived using the Asset and Health Dynamics (AHEAD) Study respondent population weights to adjust for the complex sampling design of the AHEAD survey.

${ }^{\dagger} \mathrm{P}<.05$ by the $\chi^{2}$ test for a significant association between the indicated variable and dementia category.

ADLs, activities of daily living; IADLs, instrumental activities of daily living.

percent of respondents showed evidence of cognitive impairment consistent with dementia. Of those with dementia, $43 \%$ were in the mild category, $27 \%$ were moderate, and $31 \%$ were severe. Compared to persons with normal cognitive function, those with dementia (all levels of severity) were older, more likely to be African American, unmarried, have less than a high school education, have low net worth, have more ADL and IADL difficulties, and were more likely to have a diagnosis of stroke, diabetes, heart disease, psychiatric problem, and arthritis $(P<.05$ for all comparisons). As expected, dementia severity was positively associated with the likelihood of being represented by a proxy.

\section{Incremental Weekly Hours and Yearly Cost of Informal Caregiving}

The adjusted incremental weekly hours (derived from the two-part model) and associated incremental yearly cost of informal caregiving for each level of cognitive impairment are shown in Table 2. There was a significant positive association of dementia severity and weekly hours of informal care. Those elderly with normal cognitive status received an average of 4.6 hours per week of informal care. Those with mild dementia received an additional 8.5 hours of care compared to those with normal cognition, while those with moderate dementia received an additional 17.4 hours, and those with severe dementia received an additional 41.5 hours of care $(P<.001)$.

Using the middle-range estimate of the opportunity cost of an informal caregiver's time ( $\$ 8.20$ per hour), the incremental yearly cost of caregiving attributable to dementia ranged from $\$ 3,630$ for mild dementia to $\$ 17,700$ for severe dementia. Using the more conservative low-range opportunity cost estimate ( $\$ 5.90$ per hour), the incremental yearly cost of dementia caregiving ranged from $\$ 2,610$ for mild dementia to $\$ 12,730$ for severe dementia, while the corresponding high-range estimates ( $\$ 10.80$ per hour) were $\$ 4,780$ for mild dementia and $\$ 23,310$ for severe dementia.

To assess the appropriateness of our chosen cut-off for defining dementia, we tested whether those selfrespondents with TICS scores of 9 to 11 received significantly greater hours of informal care. Using the same two-part model as for the main analysis, this "borderline" group received a similar number of weekly hours of informal care as those in the "normal" group, and significantly fewer hours of care than those in the "mild" dementia group. Similarly, respondents whose memory was rated as "good" by their proxy did not receive significantly more informal hours than those in the "normal" group (data not shown). These findings suggest that the dementia cut-offs that we used, a score of 8 on the TICS scale and a proxy assessment of "fair" memory, are both reasonable estimates of the level of cognitive impairment significant enough to impair independent functioning in everyday life.

\section{DISCUSSION}

Both clinicians and policymakers will be confronted with difficult choices regarding the allocation of health care resources as the U.S. population ages and the prevalence of dementia increases. Accurate data regarding both direct 
Table 2. Incremental Caregiving Hours and Incremental Cost of Informal Care Attributable to Dementia

\begin{tabular}{|c|c|c|c|c|}
\hline Dementia Severity & $\begin{array}{c}\text { Incremental } \\
\text { Hours per Week }^{*,}\end{array}$ & \multicolumn{3}{|c|}{ Incremental Cost per Year*, } \\
\hline Mild dementia & 8.5 & $\$ 2,610$ & $\$ 3,630$ & $\$ 4,780$ \\
\hline Moderate dementia & 17.4 & $\$ 5,340$ & $\$ 7,420$ & $\$ 9,770$ \\
\hline Severe dementia & 41.5 & $\$ 12,730$ & $\$ 17,700$ & $\$ 23,310$ \\
\hline
\end{tabular}

${ }^{*} \mathrm{P}<.001$ for comparison of hours and costs for each dementia category to that for the normal cognition category, and for comparisons between each dementia category.

${ }^{\dagger}$ Adjusted incremental weekly hours of informal care was derived from a two-part regression model that included age, race, gender, education, net worth, potential caregiver network, and chronic health conditions as independent variables.

${ }^{\ddagger}$ Adjusted incremental yearly cost of informal care was calculated by multiplying the adjusted incremental weekly hours of care by $\$ 5.90$ per hour for the low-range estimate (national $10^{\text {th }}$ percentile home health aide wage in 1998), \$8.20 per hour (mean wage) for the middle-range estimate, and $\$ 10.80$ per hour for the high-range estimate $\left(90^{\text {th }}\right.$ percentile wage), and then multiplying by 52 (weeks per year).

medical costs and informal caregiving costs are necessary to make such choices effectively. Our results extend the literature on informal caregiving for the elderly with dementia in a number of ways. Most importantly, to our knowledge, this is only the second study of informal caregiving for cognitively impaired elderly to use a population-based nationally representative sample in the United States. The first population-based study used data from 10 years prior to those of our study and did not adjust for important socioeconomic or family characteristics. ${ }^{7}$ Our estimates of caregiver time and costs may, therefore, be generalized to the noninstitutionalized U.S. population with more confidence than prior estimates, especially those derived from nonrepresentative samples. Using a population-based survey, rather than extrapolating from geographically restricted patient samples is especially important in the study of dementia because of the significant likelihood that community-dwelling elderly with cognitive impairment may not be identified and diagnosed. ${ }^{38}$

We found expected relationships between cognitive impairment and health status in that those with dementia were more likely to have ADL and IADL impairments and were also more likely to have important comorbidities, such as stroke, diabetes, heart disease, psychiatric problems, and arthritis. The higher rate of cognitive impairment among African Americans found in our analysis is likely due to a number of factors, including lower levels of education and higher rates of hypertension, diabetes, and stroke. Education has been found consistently to be an independent predictor of neuropsychological test performance, with higher education associated with better performance. ${ }^{33,39,40}$ In addition, since cognitive screening tests are culture- and language-specific, the tests may be biased against minority populations and, therefore, African Americans may be somewhat over-represented in the cognitively impaired groups in our study. ${ }^{33,41,42}$ Further study is needed to clarify the complex relationships among race, education, and cognitive impairment.

We found a striking increase in the caregiving "burden" on family members as the level of cognitive impairment worsened, from 8.5 additional hours per week for mild dementia to 41.5 additional hours per week for severe dementia. A popular guide ${ }^{43}$ for family caregivers of those with dementia compares the experience to living a "36-hour day." Similarly, we found that dementia caregiving can, in itself, be a "full-time" job. The fact that many of the caregivers working this extended week are elderly spouses (about 35\% of informal caregivers in the AHEAD survey) magnifies the importance of identifying and addressing the needs of family caregivers as the prevalence of dementia increases in the next decades. Primary care physicians, because of their sustained relationships with individuals with chronic disease, may be especially well positioned to monitor whether their patients' family members are suffering ill-effects from the potential strains of caregiving. We have previously shown that elderly women, because they are likely to receive significantly less help from family members for their own disabilities, may be especially vulnerable to the potential negative consequences associated with providing substantial dementia care. ${ }^{44}$

Given the nationally representative sample used for this analysis, an estimate of total informal caregiving costs for the cognitively impaired community-dwelling elderly age 70 years or older in the United States can be calculated. Using our definitions of dementia and the population weights for the AHEAD survey, in 1993, there were an estimated 880,000 community-dwelling elderly with mild dementia in the United States, 530,000 with moderate dementia, and 650,000 with severe dementia. The associated additional yearly cost of informal care attributable to dementia for these groups is about \$3.2 billion, \$3.9 billion, and $\$ 11.5$ billion, respectively, yielding a total additional cost of about $\$ 18.6$ billion per year. The corresponding low- and high-range caregiving cost estimates are $\$ 13.4$ billion and $\$ 24.5$ billion, respectively. To put the magnitude of informal caregiving costs for dementia in perspective, total national expenditures in 1998 for all paid home care services (not just those for dementia) were about $\$ 29$ billion, ${ }^{45}$ just 56\% greater than our mid-range estimate of informal caregiving for this single condition. 
We employed methods that likely led to conservative estimates of informal caregiving time and cost. First, only caregiving provided for help with ADLs and IADLs is included in the analysis, and infrequent help for ADLs is not captured by the AHEAD survey. The time required for caregivers to monitor and manage the behavioral problems associated with dementia (such as paranoia, hostility, and wandering), as well as the costs associated with providing shared housing and transportation, are not included in the analysis. In addition, our total cost estimate includes informal caregiving only for those elderly living in the community. While families provide significantly less informal care once an individual with dementia moves to a nursing home, some level of care from family members will likely continue. ${ }^{5,12}$ However, even using these conservative measures, and using the low-range opportunity cost estimate, the national annual cost of dementia caregiving still reaches almost $\$ 14$ billion per year. Future research should examine how the additional tasks related to dementia caregiving, such as providing supervision for safety and monitoring behavioral problems, add to the burden and costs identified in this study.

The societal cost associated with informal caregivers' time identified in this analysis may also be accompanied by significant additional effects on caregivers' work and leisure activity. For instance, in a national survey, 6\% of employed dementia caregivers reported turning down a promotion and $10 \%$ reported taking early retirement because of their caregiving responsibilities. ${ }^{46}$ About $24 \%$ of dementia caregivers reported "needing more free time or a break" from caregiving. ${ }^{46}$

In addition to the economic cost of informal caregiving, there may also be important negative health effects for dementia caregivers, including increased rates of depression $^{47}$ and even increased mortality. ${ }^{48}$ The additional costs related to such caregiver morbidity and mortality are not included in our study.

An important limitation of our analysis is that while we were able to assess level of cognitive impairment using the TICS instrument, we were unable to assign a diagnosis of dementia based on established clinical criteria, such as the Diagnostic and Statistical Manual, Fourth Edition (DSM-IV) criteria. ${ }^{16}$ However, the TICS has been shown to correlate well with other instruments (such as the $\mathrm{MMSE}^{30}$ ) used for establishing a diagnosis of dementia, and to successfully differentiate individuals with normal cognitive function from those with clinically established dementia. $^{32}$

In addition, indirect evidence suggests that our dementia classification scheme using the TICS or proxy assessment of memory performs similarly to classification methods used in prior dementia prevalence studies. A recent meta-analysis synthesizing 18 studies of $\mathrm{AD}$ prevalence estimated that there were 765,000 cases of mild $\mathrm{AD}$ among those age 70 years or older in the United States in 1995, and 1.0 million cases of moderate or severe
AD. ${ }^{1}$ Using our dementia classification, the analogous estimates for all cases of dementia (not just those resulting from $\mathrm{AD}$ ) are 880,000 cases of mild dementia, and 1.2 million cases of moderate or severe dementia.

Two potential limitations regarding the accurate reporting and measurement of caregiving hours by survey respondents or their proxies merit comment. First, it may be difficult for survey respondents to accurately assess the amount of care received for an ADL or IADL limitation if the caregiver lives with the respondent. The AHEAD survey attempts to minimize reporting differences for the caregiving hours for resident versus nonresident caregivers by identifying only caregiving that is provided specifically for limitations "due to a health problem." For instance, if a respondent reports that he/she has never typically prepared meals or gone grocery shopping, then help provided for these activities was not included as informal caregiving.

A second issue is that the perspective for reported caregiving is different for self-respondents than for proxy respondents, since self-respondents report how much caregiving they received, while proxy respondents (who are often primary caregivers) report how much caregiving they provided. To the extent that this difference in perspective results in systematic differences in the quantity of caregiving reported, biased estimates of caregiving hours may result. We performed a secondary analysis in which self-respondents and proxy respondents were analyzed separately using the same regression model as for our primary analysis. For each dementia category, caregiving hours for individuals represented by proxies were significantly greater than for self-respondents, but it is difficult to say whether this represents an overestimate of caregiving hours by proxies, an underestimate of caregiving hours by self-respondents, or that the health status of those represented by a proxy was worse than self-respondents, even after controlling for the other variables in our analysis. Since proxy informants are essential for studying those with dementia, future research should further assess how differences in the perspective of self-respondents and proxy respondents may affect caregiving results.

A number of important demographic and health system trends over the next few decades will increase the number of community-dwelling elderly with significant disabilities due to dementia and other chronic diseases. ${ }^{2,49,50}$ It will be important for future research to follow how these trends, and the public policy responses to them, affect the already significant effort expended by informal caregivers. ${ }^{51}$ Especially salient is how the U.S. long-term care system will be organized and financed to meet the increasing needs of those with dementia and their families. ${ }^{52}$ In the meantime, physicians caring for individuals with chronic disease should be aware of current public and private resources designed to provide information and support for caregivers, and make referrals to such programs when appropriate. For instance, the recently established National Family Caregiver Support Program provides 
federal funds to state and local agencies so that information, counseling, and respite opportunities will be more accessible to family caregivers. ${ }^{53}$ In addition, national organizations such as the Alzheimer's Association and the National Alliance for Caregiving can provide caregivers with information on support programs in their local communities.

This population-based analysis of informal care for those with dementia confirms the significant burden that this increasingly prevalent condition places on both families and society. This burden increases sharply as the level of cognitive impairment progresses from mild to severe. These findings, coupled with the projected explosion in the prevalence of dementia over the next 50 years, underscore the importance of including valid estimates of unpaid caregiver time when evaluating future clinical and policy interventions aimed at reducing dementia's impact on individuals, families, and society.

The Robert Wood Johnson Foundation supported Dr. Langa as a Robert Wood Johnson Clinical Scholar during part of the time that this work was performed. Additional support was provided by grants from the Michigan Alzheimer's Disease Research Center, Michigan Center on the Demography of Aging, Aetna Quality Care Research Fund, and Merck and Company, Inc. The National Institute on Aging provided funding for the Asset and Health Dynamics (AHEAD) Study (No. U01 AG09740), data from which were used in this analysis

Eric Larson, MD, MPH provided helpful comments on an early version of the manuscript and Willard G. Manning, PhD provided advice on the statistical model.

\section{REFERENCES}

1. United States General Accounting Office. Alzheimer's Disease: Estimates of Prevalence in the United States. Washington, D.C.: United States General Accounting Office; 1998.

2. Brookmeyer R, Gray S, Kawas C. Projections of Alzheimer's disease in the United States and the public health impact of delaying disease onset. Am J Public Health. 1998;88:1337-42.

3. Ernst RL, Hay JW. Economic research on Alzheimer disease: a review of the literature. Alzheimer Dis Assoc Disord. 1997;11(suppl 6): $135-45$.

4. Weinberger M, Gold DT, Divine GW, et al. Expenditures in caring for patients with dementia who live at home. Am J Public Health. 1993:83:338-41.

5. Rice DP, Fox PJ, Max W, et al. The economic burden of Alzheimer's disease care. Health Affil (Millwood). 1993;12:164-76.

6. Hu T, Huang L, Cartwright W. Evaluation of the costs of caring for the senile demented elderly: a pilot study. Gerontologist. 1986; 26:158-63.

7. Manton K, Corder L, Clark R. Estimates and projections of dementia-related service expenditures. In: Manton K, Singer B, Suzman R, eds. Forecasting the Health of Elderly Populations. New York: Springer-Verlag; 1993:207-38.

8. Ernst RL, Hay JW. The US economic and social costs of Alzheimer's disease revisited. Am J Public Health. 1994;84:1261-4.

9. Ostbye T, Crosse E. Net economic costs of dementia in Canada. Can Med Assoc J. 1994;151:1457-64.

10. Stommel M, Collins CE, Given BA. The costs of family contributions to the care of persons with dementia. Gerontologist. 1994;34: 199-205.
11. Max W, Webber P, Fox P. Alzheimer's disease: the unpaid burden of caring. J Aging Health. 1995; 7:179-99.

12. Leon J, Cheng C-K, Neumann PJ. Alzheimer's disease care: costs and potential savings. Health Aff. 1998;17:206-16.

13. Hux M, O'Brien B, Iskedjian M, Goeree R, Gagnon M, Gauthier S. Relation between severity of Alzheimer's disease and costs of caring. Can Med Assoc J. 1998;159:457-65.

14. Knapp MJ, Knopman DS, Solomon PR, Pendlebury WW, Davis CS, Gracon SI. A 30-week randomized controlled trial of high-dose tacrine in patients with Alzheimer's disease. JAMA. 1994;271: 985-91.

15. Rogers SL, Farlow MR, Doody RS, Mohs R, Friedhoff LT The Donepezil Study Group. A 24-week, double-blind, placebocontrolled trial of donepezil in patients with Alzheimer's disease. Neurology. 1998;50:136-45.

16. American Psychiatric Association. Diagnostic and Statistical Manual of Mental Disorders. Fourth Ed. Washington, D.C.: American Psychiatric Association; 1994.

17. Stone R, Cafferata GL, Sangl J. Caregivers of the frail elderly: a national profile. Gerontologist. 1987;27:616-26.

18. Kemper P. The use of formal and informal home care by the disabled elderly. Health Serv Res. 1992;27:421-51.

19. Wolf DA, Freedman V, Soldo BJ. The division of family labor: care for elderly parents. J Gerontol B Psychol Sci Soc Sci. 1997;52: $102-9$.

20. Boaz RF, Hu J. Determining the amount of help used by disabled elderly persons at home: the role of coping resources. J Gerontol B Psychol Sci Soc Sci. 1997;52:317-24.

21. Gold MR, Siegel JE, Russell LB, Weinstein MC. Cost-Effectiveness in Health and Medicine. New York: Oxford University Press; 1996.

22. Kirschstein R. Disease-specific estimates of direct and indirect costs of illness and NIH support. Washington, D.C.: National Institutes of Health; 2000. Available at: http://wwwl.od.nih.gov/ osp/ospp/ecostudies/COIreportweb.htm

23. van Dijk PT, Meulenberg OG, van de Sande HJ, Habbema JD. Falls in dementia patients. Gerontologist. 1993;33:200-4.

24. Asada T, Kariya T, Kinoshita T, et al. Predictors of fall-related injuries among community-dwelling elderly people with dementia. Age Aging. 1996;25:22-8.

25. Soldo BJ, Hurd MD, Rodgers WL, Wallace RB. Asset and health dynamics among the oldest old: an overview of the AHEAD Study. J Gerontol B Psychol Sci Soc Sci. 1997;52:1-20.

26. Norgard TM, Rodgers WL. Patterns of in-home care among elderly black and white Americans. J Gerontol B Psychol Sci Soc Sci. 1997:52:93-101.

27. Raghunathan TE, Lepkowski JM, Van Hoewyk J, Solenberger P. A Multivariate Technique for Multiply Imputing Missing Values Using a Sequence of Regression Models. Ann Arbor, Mich: Survey Research Center, Institute for Social Research; 1998.

28. Penrod JD, Kane RL, Finch MD, Kane RA. Effects of post-hospital medicare home health and informal care on patient functional status. Health Serv Res. 1998;33:513-29.

29. Folstein MF, Folstein SE, McHugh PR. "Mini-mental state". A practical method for grading the cognitive state of patients for the clinician. J Psychiatr Res. 1975;12:189-98.

30. Brandt J, Spencer M, Folstein M. The telephone interview for cognitive status. Neuropsychiatry Neuropsychol Behav Neurol. 1988;1:111-7.

31. Welsh KA, Breitner JCS, Magruder-Habib KM. Detection of dementia in the elderly using telephone screening of cognitive status. Neuropsychiatry Neuropsychol Behav Neurol. 1993;6: 103-10.

32. Plassman BL, Newman T, Welsh K, Breitner J. Properties of the telephone interview for cognitive status. Application in epidemiological and longitudinal studies. Neuropsychiatry Neuropsychol Behav Neurol. 1994;7:235-41.

33. Herzog AR, Wallace RB. Measures of cognitive functioning in 
the AHEAD Study. J Gerontol B Psychol Sci Soc Sci. 1997;52: 37-48.

34. Duan N, Manning WG, Morris CN, Newhouse JP. A comparison of alternative models for the demand for medical care. J Bus Econ Statistics. 1983;1:115-26.

35. Manning WG. The logged dependent variable, heteroscedasticity, and the retransformation problem. J Health Econ. 1998;17: 283-95.

36. Bureau of Labor Statistics. 1998 National Occupational Employment and Wage Estimates. March 2000. Available at: http:// www.bls.gov/oesnl/oes66011.htm.

37. Reference Manual STATA. Release 6.0. College Station, Tex: STATA Press; 1999.

38. Ross GW, Abbott RD, Petrovitch $\mathrm{H}$, et al. Frequency and characteristics of silent dementia among elderly JapaneseAmerican men: the Honolulu-Asia Aging Study. JAMA. 1997;277: 800-5.

39. Tschanz JT, Welsh-Bohmer KA, Skoog I, et al. Dementia diagnoses from clinical and neuropsychological data compared. The Cache County Study. Neurology. 2000;54:1290-6.

40. Scherr PA, Albert MS, Funkenstein HH, et al. Correlates of cognitive function in an elderly community population. Am J Epidemiol. 1988;128:1084-101.

41. Fillenbaum G, Heyman A, Williams K, Prosnitz B, Burchett B. Sensitivity and specificity of standardized screens of cognitive impairment and dementia among elderly black and white community residents. J Clin Epidemiol. 1990;43:651-60.

42. Teresi J, Bolden R, Cross P, Gurland B, Kleinman M, Wilder D. Item bias in cognitive screening measures: comparisons of elderly white, Afro-American, Hispanic and high and low education subgroups. J Clin Epidemiol. 1995;48:473-83.

43. Mace NL, Rabins PV. The 36-Hour Day. Baltimore, Md: The Johns Hopkins University Press; 1991.

44. Katz S, Kabeto M, Langa K. Gender disparities in the receipt of home care for elderly people with disability in the United States. JAMA. 2000;284:3022-7.

45. Levit K, Cowan C, Lazenby H, et al. Health spending in 1998: signals of change. Health Aff. 2000;19:124-32.

46. National Alliance for Caregiving. Family Caregiving in the U.S.: Findings from a National Survey. Washington, D.C: National Alliance for Caregiving; 1997.

47. Schulz R, O’Brien AT, Fleissner K. Psychiatric and physical morbidity effects of dementia caregiving: prevalence, correlates, and causes. Gerontologist. 1995;35:771-91.

48. Schulz R, Beach SR. Caregiving as a risk factor for mortality: the caregiver health effects study. JAMA. 1999;262:2215-9.

49. Hoffman C, Rice D, Sung HY. Persons with chronic conditions: their prevalence and costs. JAMA. 1996;276:1473-9.

50. Anderson GF, Hussey PS. Population aging: a comparison among industrialized countries. Health Aff. 2000;19:191-203.

51. Arno PS, Levine C, Memmott MM. The economic value of informal caregiving. Health Aff. 1999;18:182-8.

52. Merlis M. Caring for the frail elderly: an international review. Health Aff. 2000;19:141-9.

53. Administration on Aging. Family caregiving. Available at: http:// www.aoa.gov/may2001/factsheets/family-caregiving.html.

\begin{tabular}{|c||}
\hline ANNOUNCEMENT \\
You can reach JGIM on the Internet! \\
For more information \\
about submitting manuscripts to JGIM \\
or if you would like to submit a \\
Letter to the Editor or a \\
short creative writing piece \\
for possible publication in the Journal, \\
please e-mail us at \\
jgim@jhmi.edu
\end{tabular}

